\title{
Treatment and predictive factors in patients with recurrent laryngeal carcinoma: A retrospective study
}

\author{
PEIJING LI $^{1 *}$, WEIHAN HU ${ }^{1 *}$, YUAN ZHU ${ }^{2}$ and JIANJIANG LIU ${ }^{3}$ \\ ${ }^{1}$ Sun Yat-Sen University Cancer Center, State Key Laboratory of Oncology in South China, \\ Collaborative Innovation Center of Cancer Medicine, Guangzhou, Guangdong 510060; ${ }^{2}$ Department of Radiation Oncology, \\ Zhejiang Cancer Hospital, Hangzhou, Zhejiang 310022; ${ }^{3}$ Department of Radiation Oncology, \\ Shaoxing People's Hospital, Shaoxing, Zhejiang 312000, P.R. China
}

Received October 12, 2014; Accepted July 28, 2015

DOI: $10.3892 / 01.2015 .3640$

\begin{abstract}
The aim of the present retrospective analysis was to evaluate the treatment results for recurrent laryngeal cancer and to analyze the factors that may affect survival. A retrospective review was performed in 309 patients with recurrent laryngeal carcinoma, 224 of whom were treated between 1996 and 2009 at Sun Yat-Sen University Cancer Center (Guangzhou, Guangdong, China) and 85 of whom were treated between 2006 and 2013 at Zhejiang Cancer Hospital (Hangzhou, Zhejiang, China). The Kaplan-Meier method with the log-rank computation was used for the analysis of survival. The Cox proportional hazards model was applied to identify covariates that were significantly associated with overall survival. The actuarial 3-, 5- and 10-year survival rates were $68.9,53.6$ and $35.7 \%$, respectively. Univariate analysis indicated that the following factors were associated with survival: Age, smoking index, tumor grade, primary tumor site, nodal status of the primary tumor, initial $\mathrm{T}$ stage, initial Union for International Cancer Control stage, initial treatment (radiotherapy or chemotherapy), disease-free interval, eligibility for surgery and extent of recurrence. Multivariate analysis of these factors indicated that the initial $\mathrm{T}$ stage, tumor grade, nodal status, disease-free interval and eligibility for surgery were significantly associated with survival. Overall, these results indicate that initial tumor stage $\mathrm{T} 1$ or $\mathrm{T} 2$, a high differentiation rate, no initial cervical lymph node metastasis, a long disease-free interval and a strong indication for salvage surgery are good prognostic factors for the survival of patients with recurrent laryngeal carcinoma.
\end{abstract}

Correspondence to: Dr Jianjiang Liu, Department of Radiation Oncology, Shaoxing People's Hospital, 568 Zhongxing North Road, Shaoxing, Zhejiang 312000, P.R. China

E-mail: jianjiang08@163.com

*Contributed equally

Key words: laryngeal carcinoma, predictive factor, prognosis, recurrence

\section{Introduction}

Head and neck cancer (HNC) includes several cancers that originate in the nasal cavity, salivary glands, paranasal sinuses, pharynx, oral cavity and larynx (1). Approximately 42,440 new HNC cases were diagnosed and 8,390 HNC-related fatalities were reported in the United States in 2014. Laryngeal carcinoma accounts for $33.9 \%$ of all HNCs (2). Laryngeal carcinoma typically affects middle-aged men, and squamous cell carcinomas (SCCs) account for $90 \%$ of laryngeal carcinomas. Recurrence is common in laryngeal cancer. The recurrence rate in patients with T1 stage laryngeal cancer varies from 5 to $13 \%$, and with T2 stage cancer, it varies from 25 to $30 \%$ (3). As for patients with T3 and T4 stage disease, the recurrence rate is $30-50 \%$ (4-6). According to the tumor location, laryngeal carcinoma can be classified as supraglottic carcinoma, glottic carcinoma or subglottic carcinoma. Primary tumors in the subglottic area are relatively less common, and only account for 1-2\% of all laryngeal cancers (7). The majority of subglottic carcinomas are found late in the course of the disease, at which point surgery is difficult. Furthermore, the metastasis rate of anterior cervical lymph nodes and paratracheal lymph nodes is high, which contributes to a poorer prognosis and a higher post-operative recurrence rate (8). The majority of the treatment failures in cases of supraglottic laryngeal cancer are due to difficulty in the eradication of locoregional disease, particularly regional disease in the cervical lymph nodes. Recurrence most frequently occurs in the larynx, including the peristomal area, followed by the regional lymph nodes and the less commonly affected distant sites $(9,10)$.

The treatment options for recurrent laryngeal carcinoma are as follows: i) Repeated radiotherapy with or without chemotherapy, ii) salvage surgery, iii) supportive treatment or iv) palliative chemotherapy. The preferred method for the curative treatment of recurrent laryngeal cancer after failure of non-surgical (radiotherapy or chemoradiotherapy) treatment failure is surgery. Conservation surgery is feasible in approximately one-third of these recurrent cancers. Endoscopic resection using a $\mathrm{CO}_{2}$ laser or open partial laryngectomy (partial vertical, supracricoid or supraglottic laryngectomies) can also be used. A previous study showed that, compared 
with total laryngectomy, conservation surgery has a better prognosis, but it is possible that these results were caused by a selection bias, as more advanced patients had to receive a total laryngectomy (11). As for patients who have undergone surgery, a second or third surgery can also be the first choice as a radical treatment $(12,13)$. Recurrent advanced-stage cancers and those with subglottic extension should generally be treated by total laryngectomy. The use of adjuvant therapy depends on the risk factors. Radiotherapy with concurrent systemic therapy is recommended when the recurrence sites are unresectable or when the patient has not previously undergone radiotherapy, providing the patient's general condition allows it. If a patient with tumor recurrence is neither eligible for surgery nor eligible for radiotherapy, then the treatment approach is the same as that for patients with metastatic disease. The overall 5-year survival rate of patients with local recurrence who undergo salvage surgery varies greatly from 22 to $66 \%$ (14-17). One previous study has even shown the 5 -year survival rate in this patient population to be as low as $2 \%$ (4).

The present retrospective review was performed in 309 patients with recurrent laryngeal carcinoma from two institutions, who were managed with the aforementioned four treatments. This study was performed to evaluate the treatment outcomes of patients with recurrent laryngeal carcinoma and to identify potential prognostic factors that may affect survival, so as to determine appropriate treatment strategies for these patients.

\section{Materials and methods}

Characteristics of the patients and enrollment criteria. A total of 2,091 laryngeal carcinoma patients, consisting of 1,621 patients treated between 1996 and 2009 at Sun Yat-Sen University Cancer Center (Guangzhou, Guangdong, China) and 470 patients treated between 2006 and 2013 at Zhejiang Cancer Hospital (Hangzhou, Zhejiang, China), were analyzed. Of these laryngeal carcinoma patients, 2,007 patients had biopsy-proven SCC of the larynx. Of these 2,007, 309 patients (15.4\%) were diagnosed with recurrent disease. The median follow-up time was 49.5 months (range, 7-176 months). Only patients in whom the initial cancer was completely cured using radiation therapy or surgery, with or without chemotherapy, were included. Each patient's basal demographic and clinical characteristics were recorded, including smoking index, gender, age, blood type, alcohol consumption, cancer stage, histological differentiation and treatments. Patients were restaged according to the Union for International Cancer Control (UICC) 2002 cancer staging system (18), and patients with distant metastasis were excluded.

Finally, 309 patients (299 males, 96.8\%; 10 females, $3.2 \%$ ) were retrospectively analyzed. The complete clinical and pathological data are shown in Table I. Of these patients, 184 presented with supraglottic carcinoma (59.5\%), 110 with glottic carcinoma (35.6\%) and 15 with subglottic carcinoma (4.9\%). With regard to tumor stage, 97 (31.4\%) patients presented with T1 tumors, $110(35.6 \%)$ with T2 tumors, $57(18.4 \%)$ with T3 tumors and $45(14.6 \%)$ with T4 tumors. The tumors of 122 patients (39.5\%) exhibited good differentiation,
138 (44.7\%) exhibited moderate differentiation and 49 patients (15.9\%) exhibited poor differentiation. The median age of diagnosis was 59 years (range, 16-83 years).

Treatment approaches for the initial tumors. With the aid of a multidisciplinary team discussion, each patient's treatment preferences and economic status were comprehensively analyzed in order to determine the preferable treatment approach. A total of 267 patients (86.4\%) initially underwent surgery, 37 (12.0\%) underwent chemotherapy and 146 (47.2\%) underwent radiotherapy. The patients who underwent radiotherapy were divided into two groups, the radiotherapy and radiotherapy plus surgery groups (Table II). Chemotherapy and the order of treatment were not considered in the grouping, and concurrent chemoradiotherapy was regarded as radiotherapy alone. For each group, univariate analysis was used to determine whether there was a correlation between radiotherapy dose and the 5-year overall survival (OS) of patients with recurrence who underwent radiotherapy initially. The patients who underwent radiotherapy were divided again into two groups according to the median radiotherapy dose. The details of the total treatment for the primary site and the neck are as follows: i) 154 patients underwent surgery alone; ii) 95 patients underwent surgery plus 38-72 Gy of post-operative radiotherapy with or without concurrent chemotherapy; iii) 33 patients underwent radiotherapy alone with 36-74 Gy of radiation; iv) 1 patient underwent 66 Gy of radiotherapy plus surgery; and v) 26 patients were treated with combined cisplatin-based chemotherapy, including 8 who underwent surgery plus adjuvant chemotherapy, 6 who underwent neoadjuvant chemotherapy plus radiotherapy, 6 who underwent neoadjuvant chemotherapy plus surgery and 34-64 Gy of post-operative radiotherapy, 4 who underwent radiotherapy plus adjuvant chemotherapy and 2 who underwent surgery plus adjuvant chemotherapy with 68-70 Gy of post-operative radiotherapy.

Follow-up time and method. Outpatient check-ups were the main means of follow-up. Clinical examination, magnetic resonance imaging, intensive computed tomography (CT), abdominal sonography or fiberoptic endoscopy was performed every 2-3 months for the first 2 years after treatment and then every 6 months thereafter until mortality. Positron emission tomography-CT was performed if recurrent disease could not be identified or if it was suspected on routine CT. Bone scans were employed when bone metastasis was suspected. Other tests were performed at the discretion of the treating physician. The median follow-up time was 47 months (range, 7-176 months).

Statistical analysis. The Kaplan-Meier method and proportional hazards regression model were used to estimate OS and its correlation with relevant variables. Differences between groups were calculated using the log-rank test. SPSS software (version 19.0; IBM SPSS, Armonk, NY, USA) was used to calculate OS and its correlation with relevant variables. Tests of significance were performed using a two-sided value of $\mathrm{P}<0.05$. Date of recurrence was defined as the time-point at which the imaging examination suggested recurrence. Survival of recurrent patients was calculated from the date of 
Table I. Univariate analysis of the prognostic factors for 5-year OS.

\begin{tabular}{|c|c|c|c|c|c|}
\hline Prognostic factor & No. of patients & $\%$ & 5-year OS & $\chi^{2}$ & P-value \\
\hline Age, years $^{\mathrm{b}}$ & & & & 7.52 & 0.006 \\
\hline$\geq 60$ & 151 & 48.9 & 45.0 & & \\
\hline$<60$ & 158 & 51.1 & 64.5 & & \\
\hline Alcohol consumption & & & & 1.39 & 0.238 \\
\hline Yes & 175 & 42.7 & 49.7 & & \\
\hline No & 134 & 57.3 & 56.2 & & \\
\hline Grade & & & & 21.56 & $<0.001$ \\
\hline Poor & 49 & 15.9 & 23.6 & & \\
\hline Moderate & 138 & 44.7 & 55.1 & & \\
\hline Well & 122 & 39.5 & 62.2 & & \\
\hline Smoking years & & & & 0.006 & 0.939 \\
\hline$<20$ & 72 & 23.3 & 57.1 & & \\
\hline$\geq 20$ & 237 & 76.7 & 52.6 & & \\
\hline Smoking index ${ }^{c}$ & & & & 7.84 & 0.005 \\
\hline$\geq 600$ & 138 & 56.6 & 54.1 & & \\
\hline$<600$ & 122 & 43.4 & 71.1 & & \\
\hline Gender & & & & 3.48 & 0.062 \\
\hline Male & 299 & 96.8 & 53.0 & & \\
\hline Female & 10 & 3.2 & 77.8 & & \\
\hline Blood type & & & & 0.67 & 0.880 \\
\hline A & 89 & 28.8 & 57.4 & & \\
\hline B & 77 & 24.9 & 50.0 & & \\
\hline $\mathrm{AB}$ & 30 & 9.7 & 49.5 & & \\
\hline $\mathrm{O}$ & 113 & 36.6 & 51.6 & & \\
\hline Initial tumor $\mathrm{T}$ stage & & & & 42.55 & $<0.001$ \\
\hline 1 and 2 & 207 & 67.0 & 67.0 & & \\
\hline 3 and 4 & 102 & 33.0 & 28.0 & & \\
\hline Initial tumor UICC stage & & & & 44.66 & $<0.001$ \\
\hline I and II & 181 & 58.6 & 68.9 & & \\
\hline III and IV & 128 & 41.4 & 31.5 & & \\
\hline Primary site & & & & 10.47 & 0.006 \\
\hline Supraglottic & 184 & 59.5 & 60.3 & & \\
\hline Glottic & 110 & 35.6 & 40.2 & & \\
\hline Subglottic & 15 & 4.9 & 49.0 & & \\
\hline Initial nodal status & & & & 40.56 & $<0.001$ \\
\hline Negative & 231 & 74.8 & 62.8 & & \\
\hline Positive & 78 & 25.2 & 24.0 & & \\
\hline Chemotherapy for the initial tumor & & & & 14.624 & 0.016 \\
\hline Yes & 37 & 12.0 & 23.3 & & \\
\hline No & 272 & 88.0 & 57.8 & & \\
\hline Surgery for the initial tumor & & & & 0.108 & 0.742 \\
\hline Yes & 267 & 86.4 & 50.5 & & \\
\hline No & 42 & 13.6 & 54.5 & & \\
\hline Radiotherapy for the initial tumor & & & & 15.257 & $<0.001$ \\
\hline Yes & 146 & 47.2 & 40.0 & & \\
\hline No & 163 & 52.8 & 66.2 & & \\
\hline Initial treatment with radiotherapy ${ }^{\mathrm{d}}$ or surgery only & & & & 17.255 & $<0.001$ \\
\hline Radiotherapy & 42 & 13.6 & 50.5 & & \\
\hline Radiotherapy plus surgery & 104 & 33.7 & 37.1 & & \\
\hline Surgery & 163 & 52.8 & 66.5 & & \\
\hline
\end{tabular}


Table I. Continued.

\begin{tabular}{|c|c|c|c|c|c|}
\hline Prognostic factor & No. of patients & $\%$ & 5-year OS & $\chi^{2}$ & P-value \\
\hline Mode of recurrence & & & & 36.54 & $<0.001$ \\
\hline Local only & 176 & 57.0 & 61.8 & & \\
\hline Regional ( \pm local) & 104 & 33.7 & 52.1 & & \\
\hline Distant $( \pm$ regional \pm local $)$ & 29 & 9.3 & 0 & & \\
\hline Disease-free interval, months ${ }^{\mathrm{e}}$ & & & & 22.25 & $<0.001$ \\
\hline$\geq 12$ & 162 & 52.4 & 74.1 & & \\
\hline$<12$ & 147 & 47.6 & 44.0 & & \\
\hline Eligibility for surgery & & & & 47.98 & $<0.001$ \\
\hline Eligible & 211 & 68.3 & 73.0 & & \\
\hline Not eligible & 98 & 31.7 & 32.3 & & \\
\hline
\end{tabular}

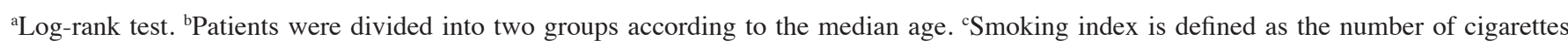
smoked per day $\mathrm{x}$ the total smoking time (years). ${ }^{\mathrm{d} C h e m o t h e r a p y}$ and the order of treatment were not considered in the grouping, and concur-

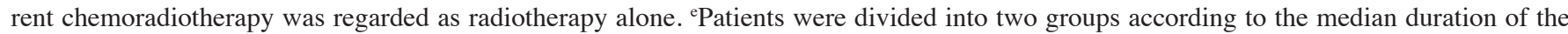
disease-free interval. OS, overall survival; UICC, Union for Internation Cancer Control.

Table II. Univariate analysis of radiotherapy dose as a prognostic factor for the 5-year OS of patients with recurrent tumors who underwent radiotherapy for the initial tumor.

\begin{tabular}{|c|c|c|c|}
\hline Factor & No. of patients & 5-year OS & P-value ${ }^{b}$ \\
\hline Initial treatment $^{\mathrm{c}}$ & & & 0.223 \\
\hline Radiotherapy alone, Gy & 42 & 50.5 & 0.772 \\
\hline$<60$ & 16 & 48.2 & \\
\hline$\geq 60$ & 26 & 51.8 & \\
\hline Surgery plus radiotherapy, Gy & 104 & 37.1 & 0.956 \\
\hline$<60$ & 41 & 36.7 & \\
\hline$\geq 60$ & 63 & 37.4 & \\
\hline
\end{tabular}

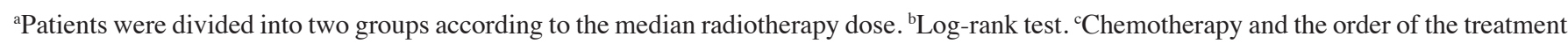
were not considered in the grouping, and concurrent chemoradiotherapy was regarded as radiotherapy alone.

recurrence to the date of mortality or to the last date when the patients were known to be alive.

Study approval. The study was approved by the Research Ethics Committee of Zhejiang Cancer Hospital and the Research Ethics Committee of the Sun Yat Sen University Cancer Center. The present study was retrospective.

\section{Results}

Sites of recurrence and relevant treatment. The median disease-free interval for the patients was 12 months (range, 3-124 months). A total of 176 patients $(57.0 \%)$ experienced local recurrence only and 21 patients $(6.8 \%)$ experienced additional regional recurrence. In 83 patients $(26.9 \%)$, the treatment for the initial tumor had failed in the regional lymph nodes only, and 22 patients $(7.1 \%)$ had isolated distant metastases. A total of 4 patients $(1.3 \%)$ developed metastases and distant regional lymph node recurrence, and 3 patients $(0.9 \%)$ developed recurrence in all of the aforementioned sites. Treatments for the recurrent tumor are indicated in Table III. The details of the treatments administered for the recurrent tumor are as follows: i) 168 patients underwent surgery alone; ii) 14 patients underwent radiotherapy alone and 34 patients underwent radiotherapy plus postoperative radiotherapy; iii) 47 patients underwent chemotherapy alone, 3 patients underwent radiotherapy plus adjuvant chemotherapy, 12 patients underwent surgery plus adjuvant chemotherapy and 2 patients underwent surgery plus postoperative radiotherapy plus adjuvant chemotherapy; iv) 29 patients underwent no active therapy.

Predictive factors for survival. Univariate analysis showed that the following factors were indicators of good survival following recurrence: Age $<60$ years $(\mathrm{P}=0.006)$, smoking index $<600(\mathrm{P}=0.005)$, high differentiation rate $(\mathrm{P}<0.001)$, subglottic cancer $(\mathrm{P}=0.005)$, initial cancer $\mathrm{T}$ stages $\mathrm{T} 1$ and T2 $(\mathrm{P}<0.001)$, initial cancer UICC stages I and II $(\mathrm{P}<0.001)$, 
Table III. Treatment for the recurrent tumor.

\begin{tabular}{lrr}
\hline Treatment & No. of patients & $\%$ \\
\hline Surgery alone & 168 & 54.4 \\
Radiation & 48 & 15.5 \\
Radiation alone & 14 & 4.5 \\
Surgery plus postoperative radiotherapy & 34 & 11.0 \\
Chemotherapy & 64 & 20.7 \\
Chemotherapy alone & 47 & 15.2 \\
Radiotherapy plus adjuvant chemotherapy & 3 & 1.0 \\
Surgery plus adjuvant chemotherapy & 12 & 3.9 \\
Surgery plus postoperative radiotherapy plus adjuvant chemotherapy & 2 & 0.6 \\
No active therapy & 29 & 9.4 \\
\hline
\end{tabular}

Table IV. Multivariate analysis of the clinicopathological factors associated with OS in recurrent laryngeal carcinoma patients.

\begin{tabular}{|c|c|c|c|}
\hline \multirow[b]{2}{*}{ Variables } & \multicolumn{2}{|c|}{ OS } & \multirow[b]{2}{*}{ P-values } \\
\hline & Odds ratio & $95 \% \mathrm{CI}$ & \\
\hline \multicolumn{4}{|l|}{ Model A } \\
\hline Grade & 1.295 & $0.997-1.682$ & 0.053 \\
\hline Smoking index & 1.152 & $0.804-1.649$ & 0.441 \\
\hline Age, years & 1.247 & $0.882-1.763$ & 0.211 \\
\hline Initial T stage & 1.755 & $1.217-2.529$ & 0.003 \\
\hline Nodal status & 1.496 & $0.998-2.243$ & 0.051 \\
\hline Primary site & 1.134 & $0.849-1.516$ & 0.395 \\
\hline Chemotherapy or no chemotherapy for the initial tumor & 1.130 & $0.964-1.326$ & 0.132 \\
\hline Radiotherapy or no radiotherapy for the initial tumor & 1.149 & $0.965-1.370$ & 0.120 \\
\hline Mode of recurrence & 1.145 & $0.885-1.481$ & 0.303 \\
\hline Eligibility for surgery & 0.460 & $0.323-0.655$ & $<0.001$ \\
\hline Disease-free interval & 0.454 & $0.319-0.646$ & $<0.001$ \\
\hline \multicolumn{4}{|l|}{ Model B ${ }^{\mathrm{a}}$} \\
\hline Age, years & 1.355 & $0.963-1.905$ & 0.081 \\
\hline Smoking index & 1.342 & $0.944-1.907$ & 0.101 \\
\hline Grade & 1.207 & $0.935-1.559$ & 0.149 \\
\hline Primary site & 1.190 & $0.892-1.588$ & 0.236 \\
\hline Initial T stage & 2.126 & $1.501-3.013$ & $<0.001$ \\
\hline Nodal status & 1.893 & $1.261-2.841$ & 0.002 \\
\hline Chemotherapy or no chemotherapy for the initial tumor & 1.063 & $0.904-1.250$ & 0.460 \\
\hline Radiotherapy or no radiotherapy for the initial tumor & 1.167 & $0.980-1.389$ & 0.083 \\
\hline \multicolumn{4}{|l|}{ Model Ca } \\
\hline Disease-free interval & 0.439 & $0.308-0.613$ & $<0.001$ \\
\hline Eligibility for surgery & 0.407 & $0.291-0.579$ & $<0.001$ \\
\hline Mode of recurrence & 1.556 & $1.223-1.977$ & $<0.001$ \\
\hline
\end{tabular}

OS, overall survival; CI, confidence interval.

negative nodal status of the initial cancer $(\mathrm{P}<0.001)$, no radiotherapy for the initial cancer $(\mathrm{P}<0.001)$, no chemotherapy for the initial cancer $(\mathrm{P}=0.016)$, disease-free interval $\geq 12$ months $(\mathrm{P}<0.001)$, only local recurrence $(\mathrm{P}<0.001)$ and eligibility for salvage surgery $(\mathrm{P}<0.001)$ (Table I). These significant factors identified by univariate analysis were analyzed by multivariate analysis using the Cox regression model (Table IV and V). Model A was composed of variables associated with the initial and recurrent cancer, while model B included only the former and model C included only 
A

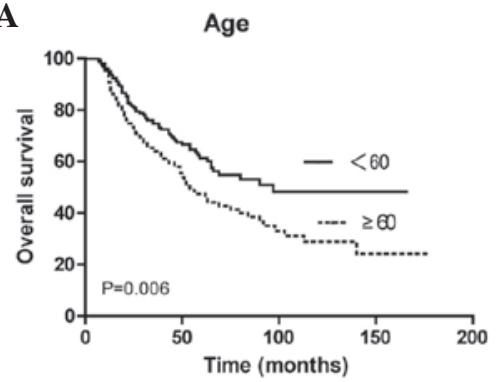

D

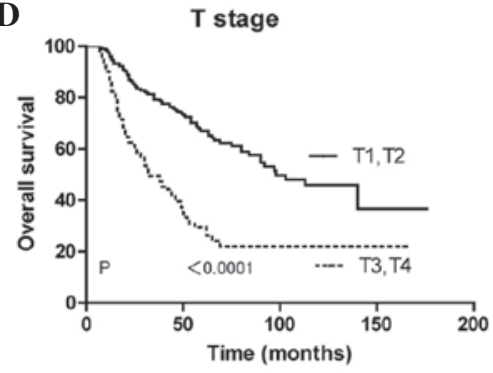

G

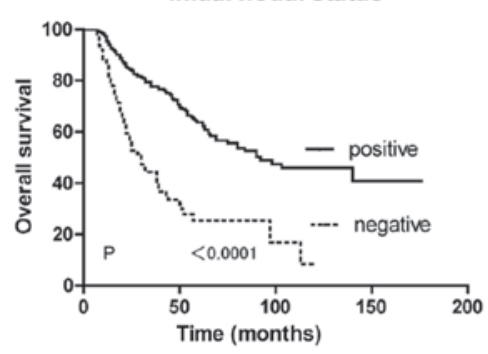

J

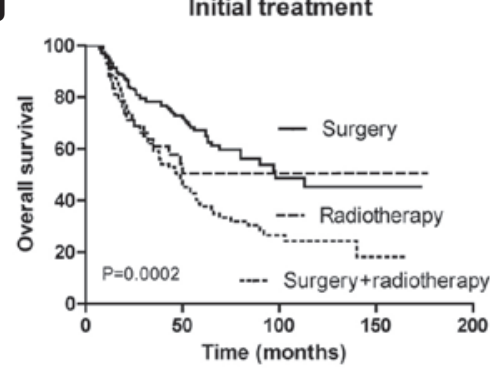

B

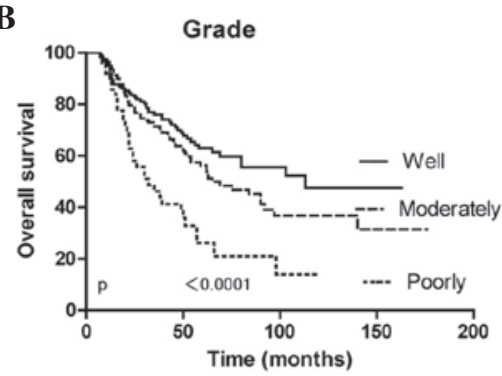

E

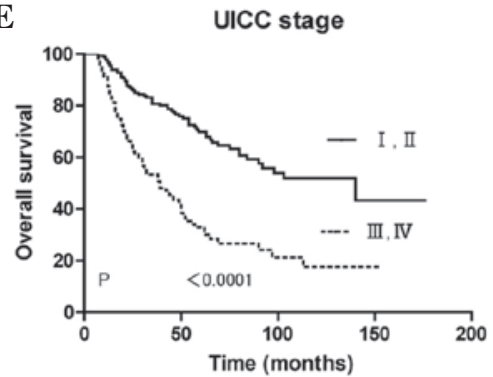

H

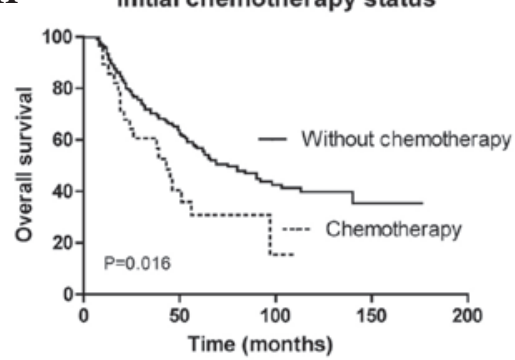

K

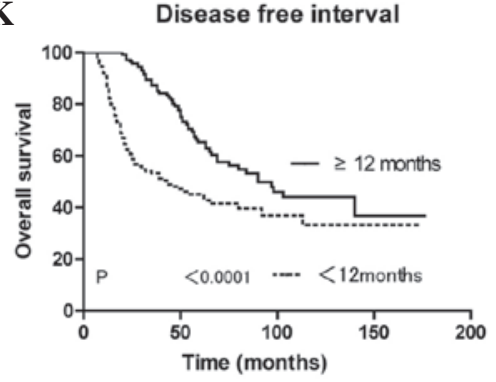

M

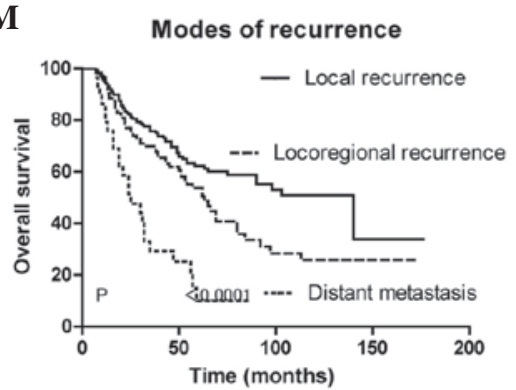

C

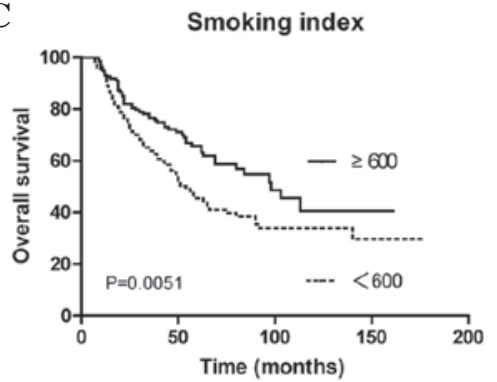

F

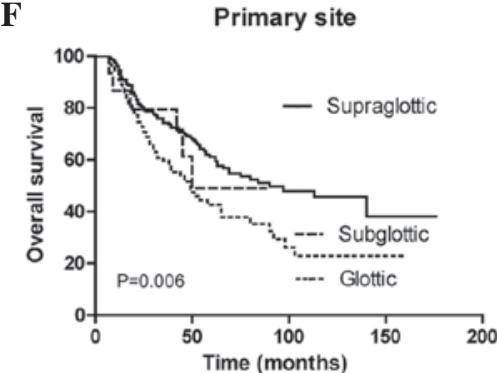

I

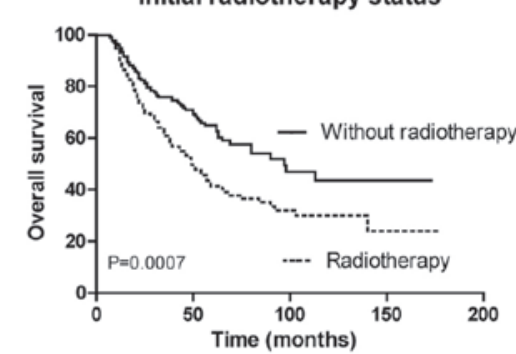

L

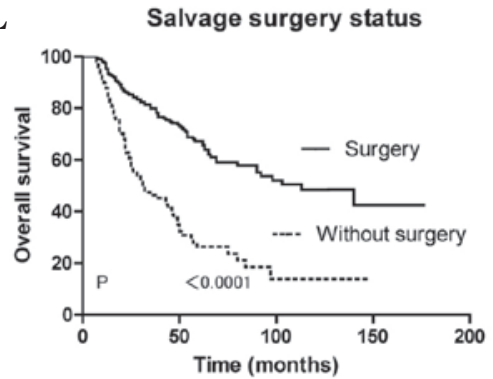

Figure 1. Survival curves according to various factors. Overall survival according to (A) age, (B) grade, (C) smoking index, (D) initial tumor T stage, (E) initial tumor UICC stage, (F) primary tumor site, (G) initial nodal status, (H) whether patients underwent chemotherapy for the initial tumor, (I) whether patients underwent radiotherapy for the initial tumor, $(\mathrm{J})$ initial different treatment, $(\mathrm{K})$ disease-free interval, (L) eligibility for surgery and (M) extent of recurrence. UICC, Union for International Cancer Control.

the latter. The initial nodal status and $\mathrm{T}$ stage were omitted in this analysis, as the initial UICC stage is based on these variables and can absolutely represent them. Model A indicated that the initial $\mathrm{T}$ stage, nodal status, disease-free interval and eligibility for salvage surgery were strong predictors of survival in the recurrent cancer patients. With regard 
Table V. Association between smoking index and initial T stage.

\begin{tabular}{lcc}
\hline Group & Number of patients & $\%$ \\
\hline Smoking index $\geq 600$ & 175 & \\
T1 and T2 stage tumor & 112 & 64.0 \\
T3 and T4 stage tumor & 63 & 36.0 \\
Smoking index <600 & 134 & \\
T1 and T2 stage tumor & 95 & 70.9 \\
T3 and T4 stage tumor & 39 & 29.1 \\
\hline
\end{tabular}

Table VI. Association between smoking index and tumor grade.

\begin{tabular}{lcc}
\hline Group & Number of patients & $\%$ \\
\hline Smoking index $\geq 600$ & 175 & \\
Well-differentiated tumor & 59 & 33.7 \\
Moderately-differentiated tumor & 85 & 48.6 \\
Poorly-differentiated tumor & 31 & 17.7 \\
Smoking index <600 & 134 & \\
Well-differentiated tumor & 62 & 46.3 \\
Moderately-differentiated tumor & 54 & 40.3 \\
Poorly-differentiated tumor & 18 & 13.4 \\
\hline
\end{tabular}

to model $\mathrm{B}$, initial $\mathrm{T}$ stage and nodal status were the only factors that predicted survival. According to model $\mathrm{C}$, all the variables included were prognostic factors.

\section{Discussion}

The present study reveals the significance of various prognostic factors for primary and recurrent laryngeal cancer, and the corresponding treatments. The 3-, 5- and 10-year survival rates of the entire patient sample were estimated to be 68.9, 53.6 and $35.7 \%$, respectively. The patients who developed local recurrence had 5-year survival rates of $61.8 \%$, which is better than the rate of $52.1 \%$ in the patients who developed regional recurrence and $0 \%$ in the patients who developed distant metastasis. These figures are in line with the results reported by Brenner et al, in which the 2- and 5-year survival rates were estimated to be 67 and 56\%, respectively (13).

Due to the natural barriers that stop the spread of cancer cells in the body and the relatively early symptoms associated with recurrence, the possibility of successfully performing salvage surgery in patients with local recurrence without damaging vital function is quite high (19). In the present study, salvage surgery resulted in a better prognosis in cases of local recurrence. Salvage surgery was performed in $68.3 \%$ of the cases of local recurrence and resulted in a 5-year survival rate of $73.0 \%$, which was much better than the $32.3 \%$ survival rate in local recurrence cases in which salvage surgery was not performed. This is similar to the results of the aforementioned study by Brenner et al (13), where $69 \%$ of the local recurrence cases received salvage surgery, with a 5-year survival rate of 76\%. However, Lacy and Piccirillo (20) reported that for $64.5 \%$ of the patients with recurrence who underwent salvage surgery, the 2-year survival rate was only $55 \%$. The reason that the present study had a better survival rate was possibly due to the fact that there were more initial early-stage patients (58.6 vs. $50.8 \%$ ). Moreover, in the study by Yuen et al (12), only $21 \%$ of the patients with local recurrence underwent salvage surgery, and the 5-year tumor-free survival rate was just $42 \%$. The reason that so few patients were suitable for salvage surgery in this study was as $97.9 \%$ patients presented with initial T3 or T4 tumors and $100 \%$ patients underwent total laryngectomy prior to recurrence.

Patients with a disease-free interval of $\geq 12$ months exhibited a $74.1 \%$ 5-year survival rate, which was significantly better than those with an interval of $<12$ months. This was also in somewhat good agreement with the figures reported by Lacy and Piccirillo (20), where the 2-year OS rate for the group with a disease-free interval of $\leq 12$ months, 2 years and $>2$ years was 33, 37 and 53\%, respectively, and Brenner et al, where the 5 -year OS rate for the group with a disease-free interval of $\leq 6$ and $>6$ months was was 44 and $70 \%$, respectively (9).

The present univariate analysis revealed that the following factors are negatively correlated with the 5-year OS rate: Age $\geq 60$ years, smoking index $\geq 600$, poor tumor grade, supraglottic and subglottic tumors, initial tumor T stages T3 and T4, initial tumor UICC stages III and IV, positive nodal status of the initial tumor, disease-free interval $<12$ months, distant metastatic disease and non-eligibility of the recurrent tumor for surgery. In addition, chemotherapy and radiotherapy for the initial tumor were found to be significant poor prognostic factors. There could be several reasons for this association. Firstly and most importantly, the effect of selection bias cannot be ruled out. Patients who initially had poor prognostic factors (poorly-differentiated cancer and more advanced UICC stage) would be more likely to receive surgery plus radiotherapy or chemotherapy with other treatment; recurrent tumors in the case of such patients would have a poorer prognosis. This probably also explains why patients who underwent surgery plus radiotherapy or chemotherapy with other treatments had a poorer 5-year OS rate than those who received surgery or radiotherapy alone (Table I). Similar results have been reported by Lacy and Piccirillo (20): The groups that received only surgery and radiotherapy for the initial tumor had a 2-year OS rate of 60 and $57 \%$, respectively, while the group that received surgery plus radiotherapy had a 2-year OS rate of only $15 \%$. Secondly, repeating the chemotherapy protocol for recurrent tumors cannot ensure effectiveness of the chemotherapy, as certain cancer cells may have drug resistance. Thirdly, if radiotherapy was already used for the initial tumor, repeat irradiation for the recurrent tumor is not feasible and at the same time, salvage surgery may also not be possible due to the acute side-effect of the radiation, particularly in patients who experienced a shorter disease-free interval.

The present study found that smoking years was not a significant prognostic factor, which is similar to the result reported by Brenner et al (13). However, the present study found that smoking index was a significant prognostic factor. This is different from the finding of Lacy and Piccirillo (20), according to which, smokers and non-smokers had a similar 2-year OS rate 
of $40 \%$. A thorough search of other relevant literature did not reveal any more findings with regard to the association between smoking and recurrent laryngeal carcinoma; however, a study on passive smoking and the incidence of laryngeal carcinoma (21) revealed that patients with advanced tumors were mostly in the environmental tobacco smoking exposure group. Therefore, the present study attempted to determine the association between smoking and initial $\mathrm{T}$ stage and tumor grade. It was found that the group with a smoking index of $\geq 600$ had a higher proportion of T3 and T4 tumors than the group with smoking index of $<600$; moreover, the former group also had a higher proportion of patients with poorly- and moderately-differentiated tumors than the latter. These values may explain why patients with recurrent tumors who had a smoking index of $\geq 600$ had poor prognostic factors.

In total, $122(39.5 \%)$ patients presented with well-differentiated tumors, 138 (44.7\%) with moderately-differentiated tumors and 49 (15.9\%) with moderately-differentiated tumors. The 5-year OS values were 62.2, 55.1 and $23.6 \%$, respectively. These were quite different from the values reported by Brenner et al (13), where the proportion of well-differentiated and moderately- and poorly-differentiated tumors was 23.2 and $76.8 \%$, respectively, with corresponding 5-year OS rates of 61 and $65 \%$, respectively. These are different from the values reported by Lacy and Piccirillo (20) too, in which the proportion of well-differentiated, moderately-differentiated, and poorly-differentiated cancer was $66.1,25.8$ and $7.3 \%$, respectively, and the corresponding 2-year OS rates were 44, 34 and $22 \%$, respectively.

Multivariate analysis showed that the 5-year OS rate was significantly associated with the initial $\mathrm{T}$ stage, grade, nodal status, disease-free interval and eligibility for surgery. This is in agreement with the results of the studies by Lacy and Piccirillo (20) and Marshak et al (9). The study by Lacy and Piccirillo revealed that initial treatment, initial TNM stage and extent of recurrence were independent prognostic factors for recurrence in laryngeal cancer. The study by Brenner et al showed that the initial tumor site, the nodal status, the extent of recurrence and its operability were the only factors that predicted survival.

The major limitation of retrospective studies is that the data collected are not originally designed for application in research. Therefore, certain factors responsible for the ultimate treatment outcome may be missing in the analysis and contribute to a bias. Thus, the conclusions from the present study should be validated in future prospective studies.

In summary, the present study found five factors to be predictors of good survival in patients with recurrent laryngeal cancer: Initial tumor T stages T1 and T2, a high differentiation rate, no cervical lymph node metastasis of the initial tumor, a disease-free interval of $\geq 12$ months and eligibility for surgery.

\section{Acknowledgements}

The authors would like to thank Dr H. Z. Wang, and Dr H. Lin for collecting data.

\section{References}

1. Argiris A, Karamouzis MV, Raben D and Ferris RL: Head and neck cancer. Lancet 371: 1695-1709, 2008.

2. Siegel R, Ma J, Zou Z and Jemal A: Cancer statistics, 2014. CA Cancer J Clin 64: 9-29, 2014.

3. Barthel SW and Esclamado RM: Primary radiation therapy for early glottic cancer. Otolaryngol Head Neck Surg 124: 35-39, 2001.

4. Answer: Yuen AP, Ho CM, Wei WI and Lam LK: Analysis of recurrence after surgical treatment of advanced laryngeal carcinoma. J Laryngol Otol 109: 1063-1067, 1995.

5 . Induction chemotherapy plus radiation compared with surgery plus radiation in patients with advanced laryngeal cancer. The department of veterans affairs laryngeal cancer study group. N Engl J Med 324: 1685-1690, 1991.

6. Lefebvre JL, Chevalier D, Luboinski B, Kirkpatrick A, Collette L and Sahmoud T: Larynx preservation in pyriform sinus cancer: Preliminary results of a European organization for research and treatment of cancer phase III trial. EORTC head and neck cancer cooperative group. J Natl Cancer Inst 88: 890-899, 1996.

7. Hanna EY: Subglottic cancer. Am J Otolaryngol 15: 322-328, 1994.

8. Strome SE, Robey TC, Devaney KO, Krause CJ and Hogikyan ND: Subglottic carcinoma: Review of a series and characterization of its patterns of spread. Ear Nose Throat J 78: 622-624, 1999.

9. Marshak G, Brenner B, Shvero J, Shapira J, Ophir D, Hochman I, Marshak G, Sulkes A and Rakowsky E: Prognostic factors for local control of early glottic cancer: The Rabin medical center retrospective study on 207 patients. Int J Radiat Oncol Biol Phys 43: 1009-1013, 1999.

10. Rübe C, Micke O, Grevers G, Rohloff R, Kaufmann H, Busch M and Willich N: Primary radiotherapy of laryngeal carcinoma. An analysis of the therapeutic results and of the relapse behavior in 283 patients. Strahlenther Onkol 173: 83-90, 1997 (In German).

11. Agra IM, Ferlito A, Takes RP, Silver CE, Olsen KD, Stoeckli SJ, Strojan P, Rodrigo JP, Gonçalves Filho J, Genden EM, et al: Diagnosis and treatment of recurrent laryngeal cancer following initial nonsurgical therapy. Head \& Neck 34: 727-735, 2012.

12. Yuen AP, Wei WI, Hui Y and Ho WK: Comprehensive analysis of pharyngeal recurrence of laryngeal carcinoma after total laryngectomy. Am J Otolaryngol 17: 380-385, 1996.

13. Brenner B, Marshak G, Sulkes A and Rakowsky E: Prognosis of patients with recurrent laryngeal carcinoma. Head Neck 23: 531-535, 2001.

14. Soo KC, Shah JP, Gopinath KS, Gerold FP, Jaques DP and Strong EW: Analysis of prognostic variables and results after supraglottic partial laryngectomy. Am J Surg 156: 301-305, 1988.

15. Bocca E, Pignataro O and Oldini C: Supraglottic laryngectomy: 30 years of experience. Ann Otol Rhinol Laryngol 92: 14-18, 1983.

16. Mittal B, Marks JE and Ogura JH: Transglottic carcinoma. Cancer 53: 151-161, 1984.

17. Davidson J, Keane T, Brown D, Freeman J, Gullane P, Irish J, Rotstein L, Pintilie M and Cummings B: Surgical salvage after radiotherapy for advanced laryngopharyngeal carcinoma. Arch Otolaryngol Head Neck Surg 123: 420-424, 1997.

18. Green FL, Page DL, Fleming ID, Fritz A, Balch CM, Haller DG and Morrow M (eds): American Joint Committee on Cancer: AJCC Cancer Staging Manual. 6th edition. Springer-Verlag, New York, NY, pp61-71, 2002.

19. Arnold DJ, Goodwin WJ, Weed DT and Civantos FJ: Treatment of recurrent and advanced stage squamous cell carcinoma of the head and neck. Semin Radiat Oncol 14: 190-195, 2004.

20. Lacy PD and Piccirillo JF: Development of a new staging system for patients with recurrent laryngeal squamous cell carcinoma. Cancer 83: 910-917, 1998

21. Mallis A, Jelastopulu E, Mastronikolis NS, Naxakis SS, Kourousis $\mathrm{C}$ and Papadas TA: Laryngeal cancer and passive smoking: the neglected factor? Eur Arch Otorhinolaryngol 268: 727-731, 2011. 